\title{
MECHANISM OF PHONATION FOR HUMAN-LIKE ROBOT (RESONANT PART OF RECTANGULAR AIR CHANNEL)
}

\author{
Yutaka TANAKA*. SON Sang Gyu**, CHOI Hung Gi** \\ *Department of Mechanical Engineering, College of Engineering \\ Hosei University \\ Koganeishi, Kajinocho, Tokyo 184, Japan \\ **Department of Mechanical Engineering , Faculty of Engineering \\ Korea University \\ Kodairashi. Ogawacho. Tokyo 187. Japan
}

\begin{abstract}
On a view point of a human-machine interface, the vocal sound is an effective medium of communication between human beings and machines both sides. Mechanism of phonation for living being is much complicated. Human phonetic organs consist of the lungs, the vocal cords, and the vocal tract. The phonemic characteristics of the vowels are determined by the frequency of the vocal cords producing fundamental sound and by the resonant behavior of the vocal tract modifying the sound to a voice. The purpose of this study is to make a human-like robot that utters sounds by a mechanical phonetic organ instead of an ordinary electrical approach. Vocal cords and a vocal tract model for mechanism of human-like phonation has been developed and Japanese five articulated vowels have been experimentally investigated by the developed resonant part.
\end{abstract}

\section{KEY WORDS}

Articulation, Air Channel, Formant, Pneumatics, Resonance, Robotics, Vocal Cords

\section{INTRODUCTION}

Mimic functions or organs for human beings have been recently studied in a field of medical engineering and bionics. Especially. there are many activities for reappearing a human vocal sound in practice. Vocal sound for human beings is much simple and important medium of communication. On a view point of a human-machine interface, the vocal sound is an effective medium of communication between human beings and machines both side

Mechanism of phonation for human beings is much complicated. The idea of modeling human vocal cords itself in attempts to produce human-like speech by replicating the physical processes has been proposed and developed by a number of authors.

Van Den Berg, et al. [9] have constructed a model of a larynx and measured the air resistance of the model. Titze [4],[5] has presented a mathematical lumpedmodel for computer simulation of human-like vocal cord motions. Flanagan, et al. [10] have directly measured voices of human talkers to determine several dynamic laryngeal functions and compared with the computer model. Mermelstein [12] models the vocal tract as a lossless acoustic tube and considers the relation between the resonant frequencies and the cross 
sectional area function. Ikeda, et al. [11] have simulated the vibration of elastic vocal cords model using a pair of membranes supported by distributed springs. In stead of replicating a human vocal organ, Yoshimoto, et al. [7] have analyzed mimic voices for the articulation mechanism of a talking bird "Mynah". Takashima, et al. [13] have studied for automatic musical performance of woodwind instruments for approach to humanoid robots.

Advances in electronics have provided new opportunities for research of an artificial voice. The vocal sound reproduced by electrical approach has been widely used around our circumstance, i.e.. rail stations or air ports. However, the electrical reproduced voice sounds unnaturally and artificially as words or sentences which are joined with the fragments of recorded human voices.

The purpose of this study is to make a human-like singing and speaking robot with a mechanical phonetic organ and air pressure control instead of electric sound. The vocal cords model made of thin rubber films has been developed in our previous study [2]. In this paper. the resonant part which mimics the vocal tract with rectangular air channels has been developed to reproduce vocal sounds. Japanese five articulated vowels have been experimentally investigated by the developed resonant part

\section{HUMAN VOICE}

\section{Human Vocal Organs}

Vocal sound for human beings is produced by an expiratory movement of many organs. A schematic view of human vocal organs is shown in Fig. 1. Human vocal organs consist of three parts; lungs, vocal cords. and a vocal tract. The lungs supply air flow to vibrate the vocal cords. The vocal cords are made of muscular

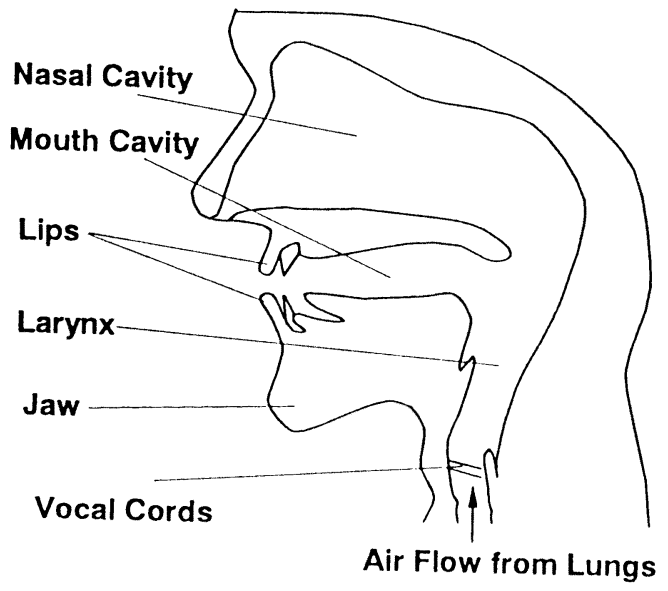

Figure 1 Human vocal organs films which have a diameter of about $40 \mathrm{~mm}$ and a thickness of about $0.5 \mathrm{~mm}$. The vocal cords are vibrated itself by the air flow and generate a fundamental sound. A male vocal sound wave has the fundamental frequency within the lower limits of about $120 \mathrm{~Hz}$. The vocal sound changes to a high pitch according to the increase of the vocal cords tension or the expiratory flow. The vocal tract includes a mouth cavity and a larynx. The fundamental sound generated by the vocal cord is resonated and changed to the vocal sound through the vocal tract. The time-dependent positions of the various articulators. e.g. tongue. lips, and jaws, are reflected in the short-time power spectrum of the voice signal, from which the formant frequencies (resonant frequency of the vocal tract) are determined.

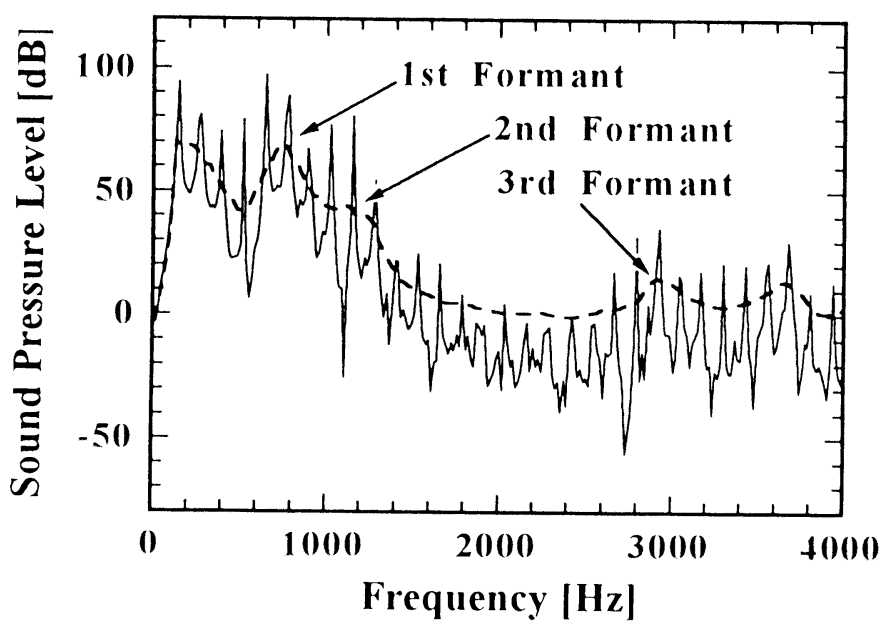

Figure 2 Frequency spectrum for vowel/a/

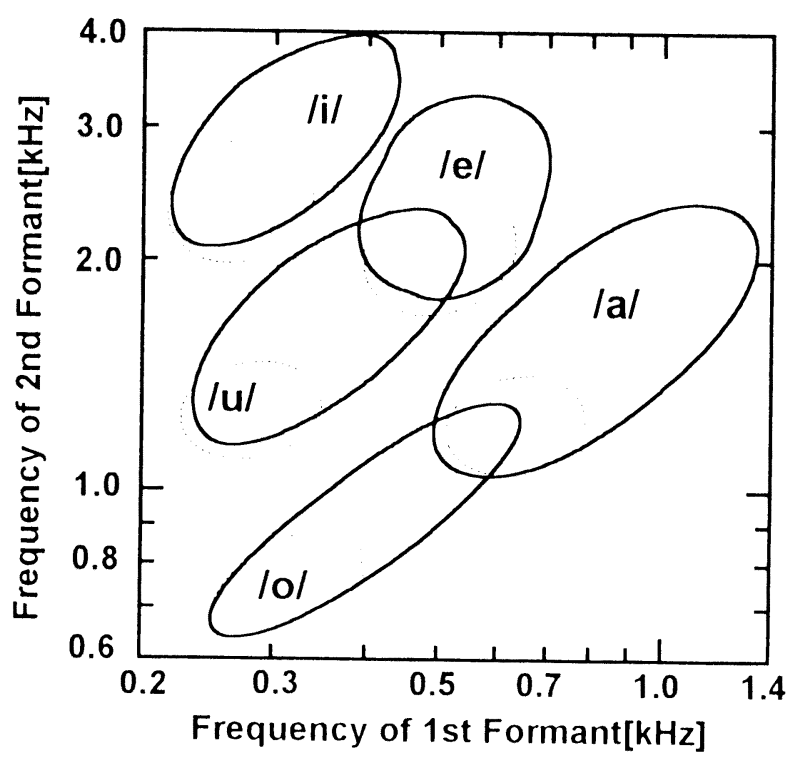

Figure 3 Correlation of 1 st vs. 2nd formant for each vowels 


\section{Acoustic Characteristics of Japanese Vowels}

The vocal sounds of Japanese five vowels depend upon the fundamental frequency of the vocal cords and each formant which are determined by the resonant frequencies of the vocal tract. Figure 2 shows a typical example of a frequency spectrum for the male vowel /a/. A solid line is calculated from the sampling data with the fast Fourier transformation (FFT). A broken line indicates the spectral envelope of the solid line. Each peak of the spectral envelope shows each formant. In turn of the lower frequency, these frequencies at the peaks are called as 1st formant, 2nd formant, and 3rd formant frequency, respectively. In the case of Japanese five vowels, each formant has the specific value of the frequency. The formant frequencies have important factors to characterize the vocal sound of each vowel. Figure 3 shows a correlation of 1st and 2nd formant frequency for each Japanese vowel. Every vowel has the specific limit of the formant frequency. The area enclosed by the solid line shows the formant data of human beings. These areas are partially distributed to overlap. As the limited area enclosed by the broken line indicates the formant data of mature males, the limits of each vowel is plotted within specific range. Acoustic characteristics for Japanese five vowels are approximately estimated by the frequency of 1 st to 3 rd formants.

\section{HUMAN-LIKE PHONETIC ROBOT}

The prototype robot consists of the compressor serving as the source of pneumatic pressure, a fundamental sound generator and a duct type resonant part.

\section{Sound producing part}

Human vocal cords are forcedly vibrated by flow motions of air caused by the sucking Bernoulli effect [9].



Figure 4 Frequency spectrum for fundamental sound of the developed sound producing part
However, the concrete behavior of vocal cords vibration has never elucidated yet. In our previous paper [2], the sound producing part which generates the fundamental sound by the pressurized air flow with the mechanism of film vibration has been developed. The sound producing part consists of an acrylic pipe and two rubber films. The acrylic pipe has an inner diameter of $46 \mathrm{~mm}$. The rubber films have a thickness of $0.5 \mathrm{~mm}$. A male vocal cords have a diameter of about $40 \mathrm{~mm}$, and a thickness of about $0.5 \mathrm{~mm}$. The dimensions of sound producing part correspond to the human vocal cords. Figure 4 shows a frequency spectrum for the fundamental sound of the developed sound producing part. The regenerated sound by the sound producing part has a fundamental frequency of about $125 \mathrm{~Hz}$. Strictly speaking, the human vocal cords does not vibrated frequently, and the human fundamental sound wave is regarded as including a fundamental frequency and the harmonic frequencies. The sound wave of a male vocal cords has the fundamental frequency of about $120 \mathrm{~Hz}$. The more the frequency of the vocal cords sound becomes high, the more the sound pressure level becomes low and reduces constantly. The frequency spectrum of the sound producing part is



Figure 5 Resonant part of rectangular air channel

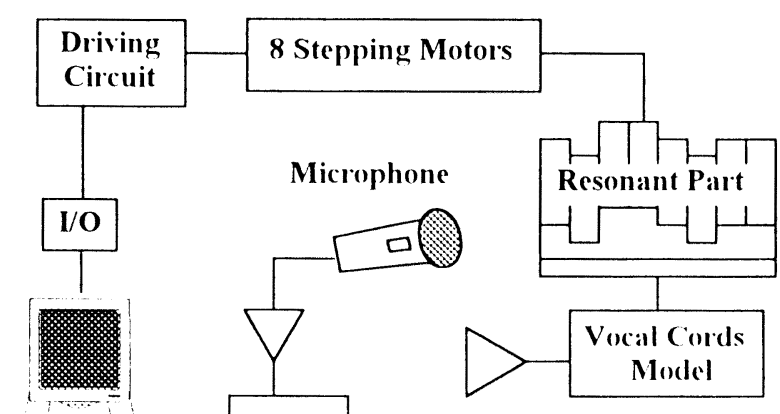

Air Supply

Figure 6 Articulation system 
similar to the spectrum of the fundamental sound for human beings. Therefor, the sound producing part model is appropriate for the sound source in this study.

\section{Resonant Part}

In general, since the diameter of the human vocal tract is smaller than the wavelength of the vocal sound, the sound is propagated with the condition of a plane wave. The acoustic characteristics of the vocal sound depend on a sectional shape of vocal tract. In this study, a vocal tract model approximated by serially connected acoustic tubes has been used for reproducing the vocal sound. The sectional areas of the respective tubes correspond to acoustic characteristics determined by the vocal tract configuration.

The number of the connected serial tubes and the formants are given as follows [3];

$$
K \geq 2 M+1
$$

where $M$ is the number of formants and $K$ is the number of tube sections. For reproducing vowels, since the 1 st to 3 rd formant may be reappeared at least, the vocal tract model is constructed by eight sections.

The developed resonant part is shown in Fig.5. The resonant part is mimicked with the rectangular air channel made of acrylic blocks. Each of these resonant parts consists of eight slidable blocks with an area of $20 \mathrm{~mm}$ wide and a slidable range of $47 \mathrm{~mm}$ long arranged alongside each other. The air channel has a total length of $160 \mathrm{~mm}$. Human phonetic organ has the length of about $160 \mathrm{~mm}$ to $180 \mathrm{~mm}$ for mature male, so the length of the resonant part nearly corresponds to the human vocal tract. Each block has a position changing mechanism that consists of a ball screw and a stepping motor. The blocks slide into the required rectangular sectional shape in conformance with the respective vocal sounds.

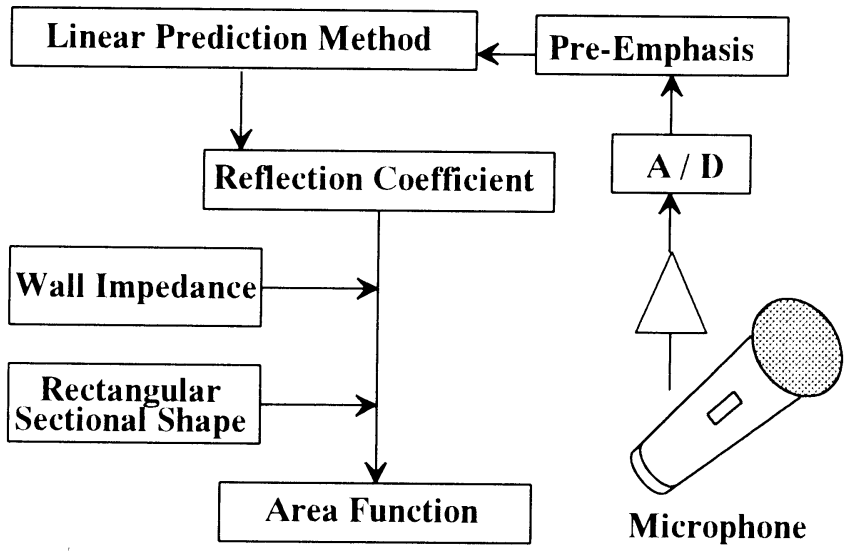

Figure 7 Process for estimation of area functions
A block diagram of an articulation system is shown in Fig.6. The air flow is supplied to the vocal cords model which generates the fundamental sound. The fundamental sound resonates in the resonant part, then the vowel sound is regenerated from the resonance characteristics. The reproduced sound is detected with a microphone and stored in the personal computer through an $\mathrm{A} / \mathrm{D}$ converter. The position of slidable blocks in the resonant part is controlled by a personal computer with $\mathrm{I} / \mathrm{O}$ port and a motor driving circuit.

\section{METHOD FOR ARTICULATION}

In this section, we consider how are the cross sectional area functions derivable from formant information. A process for estimation of the area functions for articulation in the personal computer is shown in Fig. 7.

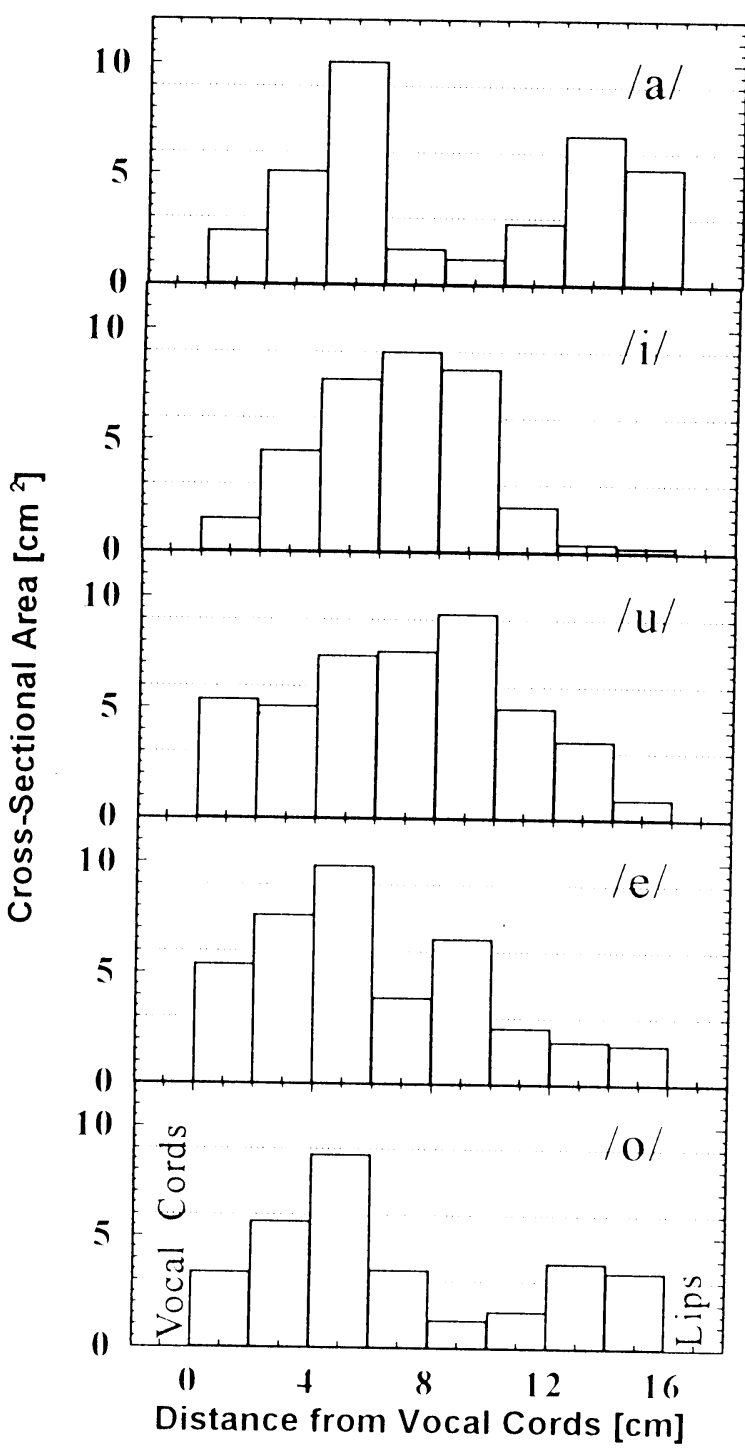

Figure 8 Area functions for Japanese vowels 


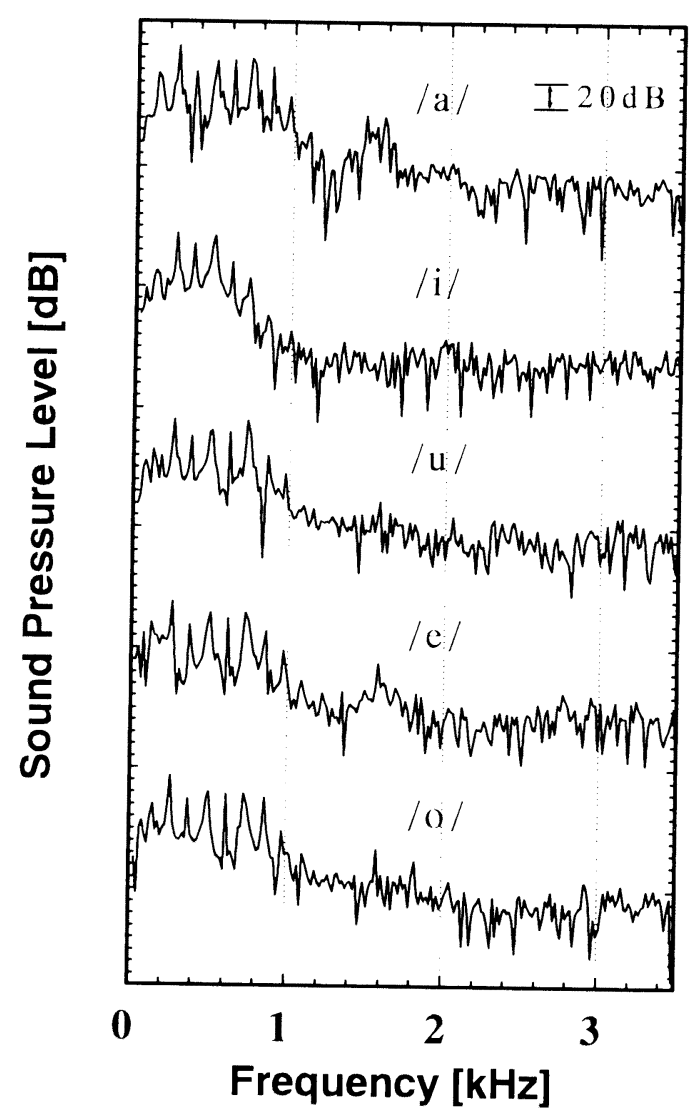

(a) Articulated sounds

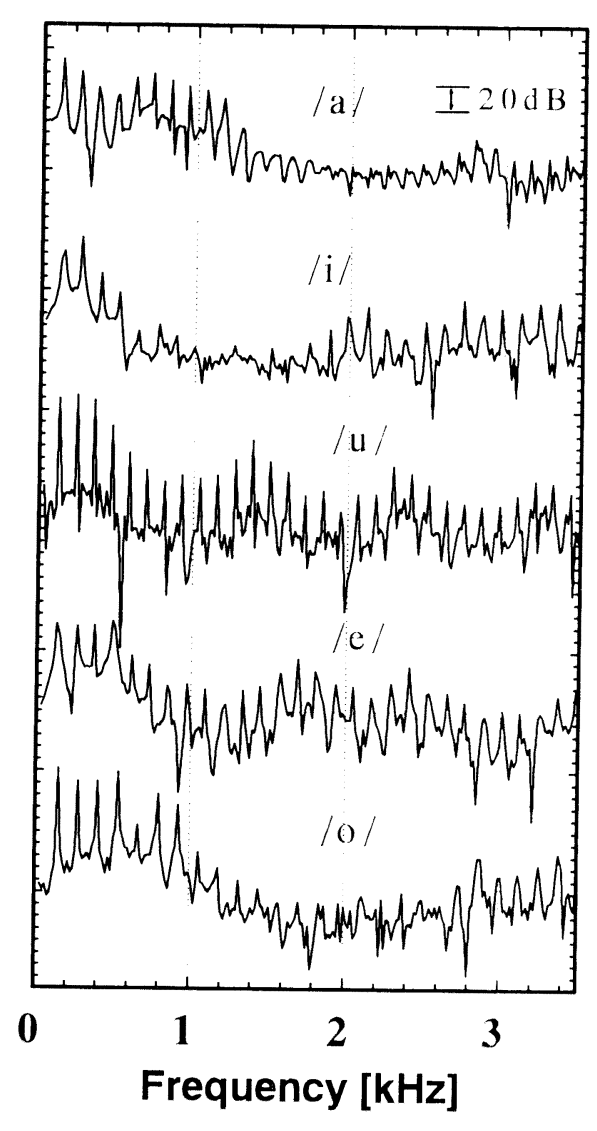

(b) Human voices

Figure 9 Frequency spectrum for each articulation vowels

The voice of the human being (mature male) is first sampled with the microphone through the A/D converter for analysis. The sampling frequency of the wave of the vowel sound is given as follows [3] :

$$
F_{s}=\frac{M C}{2 L}
$$

where $F s$ is the sampling frequency, $M$ is the number of the resonant sections, $c$ is the sound velocity, $L$ is the total length of the resonant part. Then the reflection coefficients of the sounds in the eight respective sections are calculated by the linear prediction method (LPM) [3] based on sample data of the human vowels. As the human phonetic organ is regarded as a linear all pole system. the equivalent area functions for the vowel characteristics can be estimated by the LPM. Provided that the boundary conditions at lips or teeth are given, the cross sectional area of each section is calculated in turn by the following equation:

$$
A_{m-1}=\frac{1+k_{m}}{1-k_{m}} A_{m}
$$

where $A$ is the cross sectional area and $k$ is the reflection coefficient. The area functions are rectified by considering the wall impedance of the human vocal tract and the sectional shape of the rectangular air channel.

In our experiments, the human vocal sound wave is sampled at $8.33 \mathrm{kHz}$ determined by Eq.(2). The area functions for every Japanese vowel are estimated by LPM. The examples of the estimated area function are shown in Fig.8. For example, the area function for $/ \mathrm{i} /$ is well mimicked the contraction of teeth and mouth cavity configurations. Each area function of the vowels are similar to the specific cavity configurations of human vocal tract.

\section{ARTICULATED RESULTS}

In our experiments for reproducing vowels, the area functions are calculated from the sampling data of human vowel sounds. Using the equivalent area functions, the vowel articulation is performed by the resonant part. The sampling data of the articulated vowel sound are analyzed by the FFT at 512 points. The examples of the frequency spectrum for reproduced Japanese vowels and human voices, /a/ to /o/, are shown in Fig.9 (a) and Fig.9 (b), respectively. The fundamental sound shown in Fig.4 is transformed into the articulated sound through the resonant part. The 


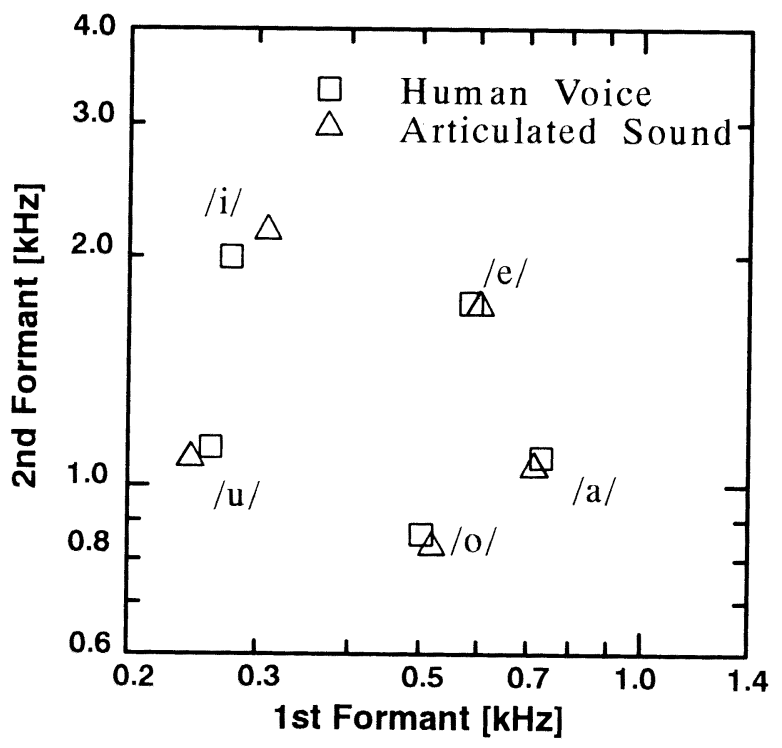

\section{Figure 10 Plot of 1st vs. 2nd formant frequency for experiments}

frequency spectrum for the articulated sound vowel, /a/ in Fig.9 indicates that the 1st, 2nd and 3rd formant frequency corresponds to $716 \mathrm{~Hz}, 1057 \mathrm{~Hz}$ and $2685 \mathrm{~Hz}$, respectively. In the case of human voice, each formant frequency is $732 \mathrm{~Hz}, 1090 \mathrm{~Hz}$ and $2913 \mathrm{~Hz}$, respectively The formant frequency of the articulated sound has nearly agreement with the formant frequency of human voice. Each formant pattern with the specific values formed by the resonant part is similar to the formant pattern of human voices. The correlation of 1 st and 2 nd formant frequency for each vowel comparing human voices with the articulated sounds is plotted in Fig.10. Every formant frequency of human beings coincides with the formant frequency of the reproduced sound. Information about higher-order formants contributes primarily to the specification of the higher spatialfrequency components. However, in our experiments, the resonant part of the eight sections can not reproduce the higer-order formants. The characteristics of the fundamental sound have also influence to the formant patterns.

\section{CONCLUSIONS}

The resonant part which mimics the human vocal tract with the rectangular air channel has been developed The fundamental sound regenerated by the vocal cords model is transformed into the articulated sound through the resonant part model. The articulated sound is similar to the human vocal sound concerning about the lower-order formant patters. Provided that the highorder formants are reproduced by the resonant part, more human-like sounds are generated. Research will be advanced on the consonants and on the nasal of the Japanese language with the aim of generating continuous sounds and realizing a human-like singing robot.

\section{ACKNOWLEDGMENTS}

This study was suggested to the authors by Prof. Nakano.K. of Shibaura Institute of Technology with his unfailing encouragement. We are also pleased to acknowledge for the experimental assistance of $\mathrm{Mr}$. Fujino, M. of Hosei University.

\section{REFERENCES}

[1] Fant,G., Acoustic Theory of Speech Wave, 1960, Mouton the Hauge.

[2] Tanaka,Y., Sato,T., Yamamoto,T., Study of A Human-Like Singing Robot (Laryngeal Model for Mechanism of Phonation), Proc. JSME Int. Conf. Advanced Mechatronics, 1993, pp59-63.

[3] Gray,A.H., Markel,J., Linear Prediction of Speech, 1976, Springer-Verlag, New-York.

[4] Titze,I.R., The Human Vocal Cords: A Mathematical Model Part I, Phonetica, 1973, 28, pp129-170.

[5] Titze,I.R., The Human Vocal Cords: A Mathematical Model Part 2, Phonetica, 1974, 29, pp1-21.

[6] Titze,I.R., On The Mechanics of Vocal-Fold Vibration, J. Acoustic Soc. Am., 1976, 60-6, pp1366-1380.

[7] Yoshimoto,C., Hirahara,T., Ifukube,T., Speech Production Process in the Mynah, J. Acoustic Soc. Am., 1978, 64-S94.

[8] Flanagan,J.L., Speech Analysis, Synthesis and Perception, 1972, Springer-Verlag.

[9] Van Den Berg, J., Zantema, J.T., Doornenbal, P., On The Air Resistance and the Bernoulli Effect of the Human Larynx, J. Acoustic Soc. Am., 1957, 29-5, pp.626-631.

[10] Flanagan,J.L., Rabiner,L.R., Christopher,D., Bock,D.E., Shipp,T., Digital Analysis of Laryngeal Control in Speech Production, J. Acoustic Soc. Am., 1976, 60-2, pp.446-455.

[11] Ikeda,T., Matsuzaki,Y., Kitagawa,T., A Model and Numerical Simulation for Speech Analysis, Preprint of Jpn. Soc. Mech. Eng. 1992, 920-7, pp.95-96.

[12] Mermelstein,P., Determination of the Vocal-Tract Shape from Measured Formant Frequencies, J. Acoustic Soc. Am., 1967, 41-5, pp. 1283-1294.

[13] Takashima,S., Shimoda,H., Automatic Performance Robot of Wind Instruments, Proc. JSME Int. Conf. Advanced Mechatronics, 1993, pp64-69. 